\title{
MANIFOLDS WITH INVOLUTION WHOSE FIXED SET HAS CODIMENSION FOUR
}

\author{
FRANK L. CAPOBIANCO
}

\begin{abstract}
Necessary and sufficient conditions are given for an $n$-manifold to be cobordant to a manifold admitting an involution whose fixed set is $(n-4)$-dimensional.
\end{abstract}

1. Introduction. Let $M^{n}$ be a closed $n$-manifold with smooth involution $T$. The fixed point set of $T$ is a disjoint union of closed submanifolds whose dimensions may vary from component to component. In this paper we study those manifolds with involution with the property that all the components of the fixed set have the same dimension. In particular, for a fixed nonnegative integer $k$ we ask: When is a closed $n$-manifold $V^{n}, n \geqslant k$, cobordant to a manifold $M^{n}$ admitting an involution $T$ whose fixed point set is $(n-k)$ dimensional? Let $J_{n}^{k}$ be the set of cobordism classes in $\Re_{n}$ which have such a representative. It is easy to see that $J_{n}^{k}$ is a subgroup of $\mathfrak{R}_{n}$ and that $J_{*}^{k}=\sum_{n=k}^{\infty} J_{n}^{k}$ is an ideal in $\Re_{*}$.

Since $\left(1, M^{n}\right)$ fixes $M^{n}, J_{*}^{0}=\mathfrak{R}_{*}$. It follows from Conner and Floyd [1, 2.1] that $J_{*}^{1}=(0)$. It also follows from Conner and Floyd [2, 27.2] that if $\left(T, M^{n}\right)$ fixes $F^{n-k}$ and $k$ is odd, then the mod 2 Euler characteristic $w_{n}\left(M^{n}\right)$ is zero. Thus for $k$ odd, $J_{*}^{k}$ is contained in the ideal of classes in $\mathfrak{N}_{*}$ with zero Euler characteristic.

R. E. Stong introduced this question [3] and proved a necessary condition that a cobordism class $\alpha$ belong to $J_{n}^{k}, k \geqslant 2$, is that $w_{1}^{n}(\alpha)=0$. For $k=2$, he proved that this condition is also sufficient.

The main result of this paper is the

Proposition. $J_{n}^{4}$ is the set of classes $\alpha$ in $\Re_{n}$ with $w_{1}^{n}(\alpha)=w_{1}^{n-4} w_{2}^{2}(\alpha)$ $=w_{1}^{n-5} s_{5}(\alpha)=0$.

I wish to express my thanks to Professor Stong for some very helpful suggestions.

2. The characteristic numbers of a class in $J_{n}^{4}$. In this section we show that the set of classes $\alpha$ in $\Re_{n}$ with $w_{1}^{n}(\alpha)=w_{1}^{n-4} w_{2}^{2}(\alpha)=w_{1}^{n-5} s_{5}(\alpha)=0$ contains $J_{n}^{4}$.

Suppose $\alpha$ belongs to $J_{n}^{4}$ and is represented by the manifold with involution

Received by the editors April 19, 1976.

AMS (MOS) subject classifications (1970). Primary 57D85; Secondary 57D90.

Copyright 1977 , American Mathematical Society 
$\left(T, M^{n}\right)$. Let $\nu^{4} \rightarrow F^{n-4}$ denote the normal bundle to the fixed point set of $T$. Let $p: \mathbf{R} P(\nu \oplus \mathbf{R}) \rightarrow F^{n-4}$ denote the projective space bundle associated to the Whitney sum of $\nu$ and a trivial line bundle. It follows from [2, 24.2] that $\alpha$ is the class of $\mathbf{R} P(\nu \oplus \mathbf{R})$, and so it suffices to show

$$
w_{1}^{n-4} w_{2}^{2}(\mathbf{R} P(\nu \oplus \mathbf{R}))=w_{1}^{n-5} s_{5}(\mathbf{R} P(\nu \oplus \mathbf{R}))=0 .
$$

Consider also the projective space bundle $q: \mathbf{R} P(\nu) \rightarrow F^{n-4}$ associated to $\nu$. If $\lambda \rightarrow \mathbf{R} P(\nu)$ is the canonical line bundle, then by [2, 28.1], $\lambda \rightarrow \mathbf{R} P(\nu)$ bords as an element of $\mathfrak{R}_{n-1}(\mathrm{BO}(1))$ and so all the characteristic numbers of $\lambda \rightarrow \mathbf{R} P(\nu)$ are zero. The characteristic numbers of

$$
\lambda \rightarrow \mathbf{R} P(\nu) \text { and } \mathbf{R} P(\nu \oplus \mathbf{R})
$$

are related by the following general facts [2]:

If $\xi^{k} \rightarrow V^{m}$ is an arbitrary smooth $k$-plane bundle and $\pi: \mathbf{R} P\left(\xi^{k}\right) \rightarrow V^{m}$ is the associated projective space bundle, let $c$ denote the characteristic class of the canonical line bundle $\lambda \rightarrow \mathbf{R} P\left(\xi^{k}\right)$. Then $H^{*}\left(\mathbf{R} P(\xi) ; \mathbf{Z}_{2}\right)$ is the free $H^{*}\left(V^{m} ; \mathbf{Z}_{2}\right)$-module via $\pi^{*}$ on the classes $1,2, \ldots, c^{k-1}$ subject to the relation $\sum_{j=0}^{k} c^{k-j} \pi^{*}\left(v_{j}\right)=0$, where $v_{j}$ is the $j$ th Whitney class of $\xi$. The total Stiefel-Whitney class of $\mathbf{R} P(\xi)$ is given by

$$
w(\mathbf{R} P(\xi))=\pi^{*}\left(w\left(V^{m}\right)\right)\left(\sum_{j=0}^{k}(1+c)^{k-j} \pi^{*}\left(v_{j}\right)\right) .
$$

We abbreviate $w_{j}\left(V^{m}\right)$ to $w_{j}$ and note explicitly that

$$
\begin{aligned}
& w_{1}(\mathbf{R} P(\xi))=\pi^{*}\left(w_{1}+v_{1}\right)+k c, \\
& w_{2}(\mathbf{R} P(\xi))=\pi^{*}\left(w_{2}+v_{1} w_{1}+v_{2}\right)+k \pi^{*}\left(w_{1}\right) c+(k-1) \pi^{*}\left(v_{1}\right) c+\left(\begin{array}{c}
k \\
2
\end{array}\right) c^{2}, \\
& s_{t}(\mathbf{R} P(\xi))=\pi^{*}\left(s_{t}\left(V^{m}\right)\right)+k c^{t}+\sum_{i=1}^{t}\left(\begin{array}{c}
t \\
i
\end{array}\right) \pi^{*}\left(s_{i}(\xi)\right) c^{t-i} .
\end{aligned}
$$

Finally we remark that the fundamental cycle $[\mathbf{R} P(\xi)]$ is related to the fundamental cycle $\left[V^{m}\right]$ by the rule $\pi_{*}\left(c^{k-1} \cap[\mathbf{R} P(\xi)]\right)=\left[V^{m}\right]$.

Let us apply these facts to our original projective space bundles $\mathbf{R} P\left(v^{4}\right)$ and $\mathbf{R} P\left(\nu^{4} \oplus \mathbf{R}\right)$. Let $c$ denote the characteristic class of $\lambda \rightarrow \mathbf{R} P(\nu)$ and $e$ the characteristic class of $\lambda \rightarrow \mathbf{R} P(\nu \oplus \mathbf{R})$. If $x \in H^{j}\left(F^{n-4} ; \mathbf{Z}_{2}\right)$ for $0 \leqslant j \leqslant n$ -4 , then the numbers $\left\langle p^{*}(x) e^{n-j},[\mathbf{R} P(\nu \oplus \mathbf{R})]\right\rangle$ and $\left\langle q^{*}(x) c^{n-j-1},[\mathbf{R} P(\nu)]\right\rangle$ are equal. Thus if $q^{*}(x) c^{n-j-1}$ represents a characteristic class of $\lambda \rightarrow \mathbf{R} P(\nu)$,

$$
\left\langle p^{*}(x) e^{n-j},[\mathbf{R} P(\nu \oplus \mathbf{R})]\right\rangle=0 .
$$

It suffices then to show $w_{1}^{n-4} w_{2}^{2}(\mathbf{R} P(\nu \oplus \mathbf{R}))$ and $w_{1}^{n-5} s_{5}(\mathbf{R} P(\nu \oplus \mathbf{R}))$ correspond to characteristic numbers of $\lambda \rightarrow \mathbf{R} P(\nu)$.

In what follows we suppress $p^{*}$ and $q^{*}$ :

LEMMA 2.1. $w_{1}^{n-4} w_{2}^{2}(\alpha)=0$. 
PRoof. Since $w_{1}(\mathbf{R} P(\nu \oplus \mathbf{R}))=w_{1}+v_{1}+e$ and $w_{2}(\mathbf{R} P(\nu \oplus \mathbf{R}))=w_{2}$ $+v_{1} w_{1}+v_{2}+w_{1} e$,

$$
\begin{aligned}
w_{1}^{n-4} w_{2}^{2}(\mathbf{R} P(\nu \oplus \mathbf{R})) & =\left(w_{1}+v_{1}+e\right)^{n-4}\left(w_{2}+v_{1} w_{1}+v_{2}+w_{1} e\right)^{2} \\
= & \left(w_{1}+v_{1}+e\right)^{n-4}\left(w_{2}^{2}+v_{1}^{2} w_{1}^{2}+v_{2}^{2}+w_{1}^{2} e^{2}\right) \\
= & \sum_{i=0}^{n-4}\left(\begin{array}{c}
n-4 \\
i
\end{array}\right)\left(w_{1}+v_{1}\right)^{i} e^{n-i-4}\left(w_{2}^{2}+v_{1}^{2} w_{1}^{2}+v_{2}^{2}+w_{1}^{2} e^{2}\right) \\
= & \sum_{i=0}^{n-6}\left(\begin{array}{c}
n-4 \\
i
\end{array}\right)\left(w_{1}+v_{1}\right)^{i}\left(w_{2}^{2}+v_{1}^{2} w_{1}^{2}+v_{2}^{2}+w_{1}^{2} e^{2}\right) e^{n-i-4},
\end{aligned}
$$

where the last equality holds because $F$ is $(n-4)$-dimensional.

On the other hand, $w_{1}(\mathbf{R} P(\nu))=w_{1}+v_{1}$ and $w_{2}(\mathbf{R} P(\nu))=w_{2}+v_{1} w_{1}+v_{2}$ $+v_{1} c$. So for each $i, 0 \leqslant i \leqslant n-6$,

$$
\begin{aligned}
& \left(w_{1}(\mathbf{R} P(\nu))\right)^{i}\left(w_{1}(\mathbf{R} P(\nu)) c+w_{2}(\mathbf{R} P(\nu))\right)^{2} c^{n-i-5} \\
& \quad=\left(w_{1}+v_{1}\right)^{i}\left(w_{2}^{2}+v_{1}^{2} w_{1}^{2}+v_{2}^{2}+w_{1}^{2} c^{2}\right) c^{n-i-5} .
\end{aligned}
$$

Therefore $w_{1}^{n-4} w_{2}^{2}(\mathbf{R} P(\nu \oplus \mathbf{R}))$ corresponds to a characteristic class of $\lambda \rightarrow \mathbf{R} P(\nu)$ and hence gives a zero number.

LEMMA 2.2. $w_{1}^{n-5} s_{5}(\alpha)=0$.

Proof. Since $s_{5}(\mathbf{R} P(\nu \oplus \mathbf{R}))=s_{5}\left(F^{n-4}\right)+s_{5}(\nu)+s_{4}(\nu) e+s_{1}(\nu) e^{4}+e^{5}$,

$$
\begin{aligned}
w_{1}^{n-5} & s_{5}(\mathbf{R} P(\nu \oplus \mathbf{R}))=\left(w_{1}+v_{1}+e\right)^{n-5} s_{5}(\mathbf{R} P(\nu \oplus \mathbf{R})) \\
= & \sum_{i=0}^{n-5}\left(\begin{array}{c}
n-5 \\
i
\end{array}\right)\left(w_{1}+v_{1}\right)^{i} e^{n-i-5} s_{5}(\mathbf{R} P(\nu \oplus \mathbf{R})) \\
= & \sum_{i=0}^{n-5}\left(\begin{array}{c}
n-5 \\
i
\end{array}\right)\left(w_{1}+v_{1}\right)^{i}\left(s_{5}\left(F^{n-4}\right)+s_{5}(\nu)+s_{4}(\nu) e+s_{1}(\nu) e^{4}+e^{5}\right) e^{n-i-5} \\
= & \sum_{i=0}^{n-6}\left(\begin{array}{c}
n-5 \\
i
\end{array}\right)\left(w_{1}+v_{1}\right)^{i}\left(s_{5}\left(F^{n-5}\right)+s_{5}(\nu)+s_{4}(\nu) e+s_{1}(\nu) e^{4}+e^{5}\right) e^{n-i-5} \\
& +\left(w_{1}+v_{1}\right)^{n-5} s_{i}(\nu) e^{4}+\left(w_{1}+v_{1}\right)^{n-5} e^{5},
\end{aligned}
$$

where the last equality holds because $F$ is $(n-4)$-dimensional. But

$$
\left(w_{1}+v_{1}\right)^{n-5} e^{5}=\left(w_{1}+v_{1}\right)^{n-5} v_{1} e^{4}=\left(w_{1}+v_{1}\right)^{n-5} s_{1}(v) e^{4},
$$




$$
\begin{aligned}
w_{1}^{n-5} s_{5}(\mathbf{R} P(\nu \oplus \mathbf{R}))=\sum_{i=0}^{n-6}\left(\begin{array}{c}
n-5 \\
i
\end{array}\right)\left(w_{1}+v_{1}\right)^{i} & \\
& \cdot\left(s_{5}\left(F^{n-4}\right)+s_{5}(\nu)+s_{4}(\nu) e+s_{1}(\nu) e^{4}+e^{5}\right) e^{n-i-5} .
\end{aligned}
$$

On the other hand, $s_{5}(\mathbf{R} P(\nu))=s_{5}\left(F^{n-4}\right)+s_{5}(\nu)+s_{4}(\nu) c+s_{1}(\nu) c^{4}$. So for each $i, 0 \leqslant i \leqslant n-6$,

$$
\begin{aligned}
\left(w_{1}(\mathbf{R} P(\nu))\right)^{i} & \left(s_{5}(\mathbf{R} P(\nu))+c^{5}\right) c^{n-i-6} \\
& =\left(w_{1}+v_{1}\right)^{i}\left(s_{5}\left(F^{n-4}\right)+s_{5}(\nu)+s_{4}(\nu) c+s_{1}(\nu) c^{4}+c^{5}\right) c^{n-i-6} .
\end{aligned}
$$

Therefore $w_{1}^{n-5} s_{5}(\mathbf{R} P(\nu \oplus \mathbf{R}))$ corresponds to a characteristic class of $\lambda \rightarrow \mathbf{R} P(\nu)$ and, hence, gives a zero number.

3. A generating set for $J_{*}^{4}$. In this section we show that $J_{*}^{4}$ contains certain classes which comprise a generating set for the set of classes $\alpha$ in $\mathfrak{R}_{n}$ with $w_{1}^{n}(\alpha)=w_{1}^{n-4} w_{2}^{2}(\alpha)=w_{1}^{n-5} s_{5}(\alpha)=0$.

Let $V^{n-7}$ be a closed manifold and let $\xi$ and $\eta$ be two smooth 4-plane bundles over $V^{n-7}$. The total space $\mathbf{R} P(\xi \oplus \eta)$ of the associated projective space bundle of the Whitney sum $\xi \oplus \eta \rightarrow V^{n-7}$ is an $n$-dimensional manifold, with involution induced by multiplication by -1 in the fibers of $\xi$ and by 1 in the fibers of $\eta$. The fixed point set of this involution is $\mathbf{R} P(\xi) \cup \mathbf{R} P(\eta)$, which has codimension 4 . Therefore to show that a cobordism class $\alpha$ belongs to $J_{n}^{4}$ it suffices to show $\alpha$ may be represented as the total space of such a bundle.

Toward this purpose recall the Stong manifolds from [3]. Let $\left(n_{1}, \ldots, n_{8}\right)$ be a sequence of nonnegative integers such that $n_{1}+\cdots+n_{8}=n-7$. Let $p: \mathbf{R} P\left(n_{1}, \ldots, n_{8}\right) \rightarrow \mathbf{R} P\left(n_{1}\right) \times \cdots \times \mathbf{R} P\left(n_{8}\right)$ be the projective space bundle associated to $\lambda_{1} \oplus \cdots \oplus \lambda_{8} \rightarrow \mathbf{R} P\left(n_{1}\right) \times \cdots \times \mathbf{R} P\left(n_{8}\right)$, where $\lambda_{i}$ is the pullback of the canonical line bundle over the $i$ th factor. Letting $\xi$ be $\lambda_{1} \oplus \cdots$ $\oplus \lambda_{4}$ and $\eta$ be $\lambda_{5} \oplus \cdots \oplus \lambda_{8}$, we see that the class of $\mathbf{R} P\left(n_{1}, \ldots, n_{8}\right)$ belongs to $J_{n}^{4}$. A useful property of these manifolds is that the class of $\mathbf{R} P\left(n_{1}, \ldots, n_{8}\right)$ is indecomposable in $\mathfrak{R}_{*}$ if and only if $\left(\begin{array}{c}n-1 \\ n_{1}\end{array}\right)+\cdots+\left(\begin{array}{c}n-1 \\ n_{8}\end{array}\right)$ is odd.

LEMMA 3.1. If $n \geqslant 9, n$ not of the form $2^{r}$ or $2^{r}-1$, then $J_{n}^{4}$ contains an indecomposable class.

Proof. By the discussion preceding this lemma, it suffices to exhibit for each $n \geqslant 9, n$ not of the form $2^{r}$ or $2^{r}-1$, a partition $\left(n_{1}, \ldots, n_{8}\right)$ of $n-7$ such that $\mathbf{R} P\left(n_{1}, \ldots, n_{8}\right)$ is indecomposable. If $\left(\begin{array}{c}n-1 \\ n-7\end{array}\right)=0 \bmod 2$, then

$$
\mathbf{R} P(n-7, \underbrace{0, \ldots, 0}_{7})
$$

is as required. Suppose $\left(\begin{array}{c}n-1 \\ n-7\end{array}\right)=1 \bmod 2$. Since $\left(\begin{array}{c}n-1 \\ n-7\end{array}\right)=\left(\begin{array}{c}n-1 \\ 6\end{array}\right)$, the dyadic expansion of $n-1$, say $2^{r_{1}}+\cdots+2^{r_{t}}$ for $r_{1}>\cdots>r_{t} \geqslant 0$, contains $2^{2}$ and 
2 as terms. Since $n \geqslant 9$ and $n$ is not of the form $2^{r}$ or $2^{r}-1$, there exists an integer $i, 1 \leqslant i \leqslant t-2$, such that $r_{i}>r_{i+1}+1$. Then

$$
\mathbf{R} P(2^{r_{1}}+\cdots+2^{r_{i}}-2,2^{r_{i+1}}+\cdots+2^{r_{t}}-6,2, \underbrace{0, \ldots, 0}_{5})
$$

gives the required class.

LEMMA 3.2. If $n=2^{r+1}, r \geqslant 2$, there exists a class $\alpha$ in $J_{n}^{4}$ such that $w_{2}^{2^{r}}(\alpha)=1$.

Proof. Suppose $r \geqslant 3$. By the discussion preceding 3.1,

$$
\mathbf{R} P(n-8,1, \underbrace{0, \ldots, 0}_{6})
$$

represents an element of $J_{n}^{4}$. From $\S 2$ we see that

Hence,

$$
w_{2}(\mathbf{R} P(n-8,1, \underbrace{0, \ldots, 0}_{6}))=p^{*}\left(w_{1}^{2}\left(\lambda_{1}\right)\right)+p^{*}\left(w_{1}\left(\lambda_{1}\right)+w_{1}\left(\lambda_{2}\right)\right) c .
$$

$$
w_{2}^{2^{r}}(\mathbf{R} P(n-8,1, \underbrace{0, \ldots, 0}_{6}))=p^{*}\left(w_{1}^{2^{r}}\left(\lambda_{1}\right)\right) c^{2^{r}}=p^{*}\left(w_{1}^{n-8}\left(\lambda_{1}\right) w_{1}\left(\lambda_{2}\right)\right) c^{7}=1
$$

when evaluated on $[\mathbf{R} P(n-8,1,0, \ldots, 0)]$ (6 zeros). For $r=2$, conjugation on $\mathbf{C} P(4)$ fixes $\mathbf{R} P(4)$ and $w_{2}^{4}(\mathbf{C} P(4))=1$.

Following [4], $\mathfrak{N}_{*}$ is a $\mathbf{Z}_{2}$-polynomial algebra on a set of generators, one in each positive dimension not of the form $2^{r}-1$; furthermore, $\alpha \in \mathfrak{N}_{n}$ is indecomposable in $\mathfrak{N}_{*}$ and hence an acceptable choice of generator if and only if $s_{n}(\alpha)=1$. For instance, we may choose generators $x_{n}$ as follows. If $n \geqslant 9, n \neq 2^{r}$, let $x_{n}$ be as in 3.1 ; let $x_{2^{r}}$ be the class of $\mathbf{R} P\left(2^{r}\right)$; let $x_{5}$ be the class of $\mathbf{R} P(2,0,0,0)$; and let $x_{6}$ be the class of $\mathbf{R} P(6) \cup \mathbf{R} P(2) \times \mathbf{R} P(4)$.

Let $I_{*}$ be the ideal of classes $\alpha$ in $\Re_{*}$ with $w_{1}^{m}(\alpha)=0$. Stong's result is that $I_{*} \supset J_{*}^{k}, k \geqslant 2$, and $I_{*}=J_{*}^{2}$. Thus provided $m \neq 2^{r}$, the generators $x_{m}$ we have chosen all belong to $I_{m}$. Furthermore, since $w_{1}^{2^{r}}(\alpha)=s_{2^{r}}(\alpha), I_{2^{r}}$ cannot contain an indecomposable, although $x_{2^{r}}^{2}$ does belong to $I_{2^{r+1}}$. Therefore, $I_{m}$ contains all products of generators for $\mathfrak{R}_{*}$ except those of the form $x_{2^{n}} \cdots x_{2^{n}}$, $r_{1}>\cdots>r_{t} \geqslant 1$, where $m=2^{r_{1}}+\cdots+2^{r_{t}}$.

From $\S 2$, the set of classes $\alpha$ in $\Re_{n}$ with $w_{1}^{n}(\alpha)=w_{1}^{n-4} w_{2}^{2}(\alpha)=w_{1}^{n-5} s_{5}(\alpha)$ $=0$ contains $J_{n}^{4}$. Note that $J_{*}^{4} \supset J_{*}^{2} \cdot J_{*}^{2}=I_{*} \cdot I_{*}$. If $m=2^{r_{1}}+\cdots+2^{r_{t}}, r_{1}$ $>\cdots>r_{t} \geqslant 1$, and $r>1$, the numbers $w_{1}^{m+2^{r+1}-4} w_{2}^{2}$ and $w_{1}^{m+2^{r+1}-5} s_{5}$ both vanish on $x_{2^{r}}^{2} x_{2^{n}} \cdots x_{2^{n}}$. Hence, the numbers $w_{1}^{n-4} w_{2}^{2}$ and $w_{1}^{n-5} s_{5}$ vanish on all products of generators for $\mathfrak{N}_{*}$ contained in $I_{*}$ except those of the form $x_{2}^{2} x_{2^{i}} \cdots x_{2^{n}}, x_{5} x_{2^{i}} \cdots x_{2^{n}}$, and $x_{6} x_{2^{\eta}} \cdots x_{2^{r}}$. By 3.2, $J_{2^{r+1}}^{4}$ contains a class $\alpha$ such that $s_{2^{r}, 2^{r}}(\alpha)=w_{2}^{2^{r}}(\alpha)=1$. Thus $\alpha=x_{2^{r}}^{2}$ modulo the ideal generated by lower dimensional classes of $J_{*}^{4}$. Therefore $J_{*}^{4}$ contains all products of generators for $\mathfrak{N}_{*}$ contained in $I_{*}$, except those of the form $x_{2}^{2} x_{2^{1}} \cdots x_{2^{n}}$, $x_{5} x_{2^{n}} \cdots x_{2^{n}}$, and $x_{6} x_{2^{n}} \cdots x_{2^{n}}$, and we have proven the 
Proposition. $J_{n}^{4}$ is the set of classes $\alpha$ in $\mathfrak{N}_{n}$ with $w_{1}^{n}(\alpha)=w_{1}^{n-4} w_{2}^{2}(\alpha)$ $=w_{1}^{n-5} s_{5}(\alpha)=0$.

\section{REFERENCES}

1. P. E. Conner and E. E. Floyd, Fibering within a cobordism class, Michigan Math. J. 12 (1965), 33-47. MR 31 \#4038.

2. - Differentiable periodic maps, Academic Press, New York; Springer-Verlag, Berlin, 1964. MR 31 \# 750.

3. R. E. Stong, On fibering of cobordism classes, Trans. Amer. Math. Soc. 178 (1973), 431-447. MR 47 \#282.

4. R. Thom, Quelques propriétés globales des variétés différentiables, Comment. Math. Helv. 28 (1954), 17-86. MR 15, 890.

Department of Mathematics, University of Wisconsin, Madison, Wisconsin 53706 